FACTA UNIVERSITATIS

Series: Mechanical Engineering Vol. 14, N ${ }^{\mathrm{o}}$ 1, 2016, pp. 37 - 53

Original scientific paper

\title{
MU-SYNTHESIS BASED ACTIVE ROBUST VIBRATION CONTROL OF AN MRI INLET
}

\author{
UDC 681.5
}

\begin{abstract}
Atta Oveisi, Tamara Nestorović
Mechanics of Adaptive Systems, Institute of Computational Engineering, Ruhr University Bochum, Germany.
\end{abstract}

\begin{abstract}
In this paper, a robust control technique based on $\mu$-synthesis is employed in order to investigate the vibration control of a funnel-shaped structure that is used as the inlet of a magnetic resonance imaging (MRI) device. MRI devices are widely subjected to the vibration of the magnetic gradient coil which then propagates to acoustic noise and leads to a series of clinical and mechanical problems. In order to address this issue and as a part of noise cancellation study in MRI devices, distributed piezo-transducers are bounded on the top surface of the funnel as functional sensor/actuator modules. Then, a reduced order linear time-invariant (LTI) model of the piezolaminated structure in the state-space representation is estimated by means of a predictive error minimization (PEM) algorithm as a subspace identification method based on the trust-region-reflective technique. The reduced order model is expanded by the introduction of appropriate frequency-dependent weighting functions that address the unmodeled dynamics and the augmented multiplicative modeling uncertainties of the system. Then, the standard D-K iteration algorithm as an output-feedback control method is used based on the nominal model with the subordinate uncertainty elements from the previous step. Finally, the proposed control system implemented experimentally on the real structure is to evaluate the robust vibration attenuation performance of the closed-loop system.
\end{abstract}

Key Words: $\mu$-synthesis, MRI Scanning Device, Robust Vibration Control, Subspace System Identification

Received February 2, 2016 / Accepted March 15, 2016

Corresponding author: Atta Oveisi

Ruhr-Universität Bochum, Universitätsstr. 150, D-44801 Bochum, Germany

E-mail: atta.oveisi@rub.de 


\section{INTRODUCTION}

MRI is an influential method that is broadly used in medical examinations and hospital clinics. The current research in the MRI technology development is mostly concentrated on improving high static magnetic field strength while keeping the speed of the current switching of the gradient coil at considerable levels. This results in a higher quality of scanning concerning the internal organs of the patient [1]. A key drawback in the development of these medical instruments is identified to be the acoustic noise that is propagated within the operational scanning [2]. One of the sources of the acoustic noise that is reported by Mechefske et al. is the gradient coil [3]. The gradient coil is used to propagate measured variations in the 3D magnetic field which permits localized image slices [3, 4]. A high acoustical noise level can engender heightened anxiety, noise-induced hearing loss, and possible permanent hearing impairment $[5,6]$. The vibration of the gradient coil can also affect the image quality and resolution [7]. McJury et al. studied impair communication problems between the patient and the hospital staff due to the acoustic noise generated by MRI devices and proposed an active noise control (ANC) technique based on the generation of destructive anti-phase interference with MRI noise [8]. Cho et al. investigated the functionality of the visual and cortex motor of the GE Signa 1.5-T EPI commercial MRI and KAIS 2.0-T research MRI devices in the presence of the sound noise due to gradient pulsing. They identified the typical behavior of the noise signal that is induced by the scanner [9]. Price et al. studied the acoustic noise of various MRI devices with a magnetic field strength range of 0.2-3 $\mathrm{T}$ and measured the noise level between 82.5-118.4 $\mathrm{dB}$ [10]. Edelstein et al. studied the acoustic noise due to the vibrations of the gradient assembly and showed the primary effect of the eddy-current-induced vibration of cryostat inner bore and the RF-body-coil in a $1.5 \mathrm{~T}$ cylindrical MRI scanner. They reduced the noise level that is transmitted through the vibration of the structure by blocking the signal pathways carrying the noise to the region of interest [11]. Mechefske et al. proposed the design of a passive acoustic liner and the gradient coil in order to suppress the noise level for the 4-T Varian/Siemens Unity INOVA whole-body MRI system [12]. Active vibration control as an effective solution can be employed to block the pathways that carry and transmit the noise in the whole MRI structure [13]. Mechefske and Shao considered the acoustic sound radiated from the MRI device under the Lorentz forces and validated the results with the boundary element method (BEM) [14]. Pribil et al. compared the spectral properties of an acoustical noise which is produced by the mechanical vibration of the gradient coils during the scanning in the open-air MRI equipment working up to $0.2 \mathrm{~T}$ [15].

The MRI shell is subjected to the excitation of the uninterrupted pulse Lorentz force of the coils. The study of MRI gradient coil relating to the sound noise was first conducted by Hurwitz et al. at the very basic level [16]. Qiu and Tani tackled the active vibration suppression due to the Lorentz force between the pulse current applied to the coil and to the main magnetic field of the circular cylindrical shell that are equipped with MRI devices [17]. Yao et al. conducted a comprehensive investigation of the vibration properties of the coil using the finite element approach (FEA) and experimental testing [1]. Nestorović et al. implemented an optimal LQ tracking controller with additional dynamics for vibration suppression of the funnel-shaped shell MRI structure [18]. They designed an additional model reference adaptive controller in order to compare the performance of the LQ controller experimentally [19]. Jaensch and Lamperth designed a micro-positioning stabilizing structure for open pre-polarization MRI scanner with piezoelectric actuators by 
means of PID controller [20]. Roozen et al. employed seismic masses which are actuated utilizing piezo-transducers by aiming at impacting the response of the gradient coil in order to actively control the vibration [21]. The application of hybrid control system consisting of both the feedback and feedforward control channels in reducing the acoustic noise of 4-T Varian Unity INOVA MRI scanner is studied by Li et al. [22].

In all of the aforementioned studies, the vibration and acoustic noise cancellation are carried out by passive methods, model-free controllers, or a deterministic model-based control approach. Although the model-free control methods are easy to implement, they are mostly limited in the performance especially due to a large working frequency of the MRI scanners and low order dynamics of the controller [23, 24]. In addition, the active control methods that are previously carried out are based on a deterministic reduced order models that are obtained by experimental-, FEM-, and BEM-based identification techniques. However, due to the limitations of the identification methods [25], the obtained model is subjected to modeling uncertainties which should be addressed by the controller. To the best knowledge of the authors of this article, no research is conducted to investigate the vibration suppression excellence by means of robust control methods. The rest of the paper is organized as follows: in section 2, the experimental configuration of the multi-input/multioutput (MIMO) structure that is used in this study for modeling and control design purposes is introduced. Then, a short overview of the identification method which is used in this paper is presented in section 3. The interested reader can refer to [26-29]. The robust output feedback control synthesis for the reduced order model that includes the input weighting functions and uncertain elements is proposed in section 4. Finally, the experimental implementation and the numerical simulation results are presented in section 5 .

\section{EXPERIMENTAL SETUP}

Since the ultimate goal of this research is vibration suppression, distributed piezotransducers are bounded on the top surface of the funnel as depicted in Fig. 1. In order to address the actuator/sensor placement, the results are used based on the research conducted by DLR Braunschweig [30].

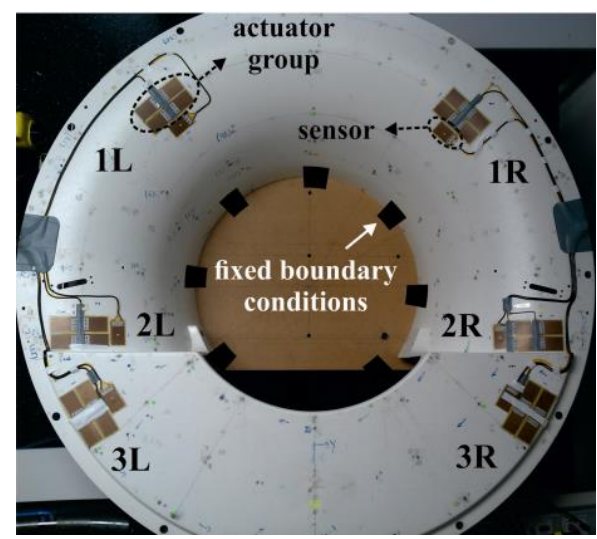

Fig. 1 Distribution of the piezo-transducers on the surface of funnel 
The structure consists of the funnel-shaped MRI inlet and six groups of sensor/actuator elements $\left(L_{1}, L_{2}, L_{3}, R_{1}, R_{2}\right.$, and $\left.R_{3}\right)$. Each group of transducers consists of four individual paired actuator-patches and one sensor element (PZT film Sonox P53). Each piezotransducer is embedded in a polymer matrix for electrical insulation and it has the overall length, width, and thickness of 50, 25, and $0.2 \mathrm{~mm}$, respectively. In order to reinforce the actuation power of the function modules, the second set of actuators are bounded on the top of the first set. The base of the funnel is fixed on a vibration isolation table with a set of screws (see Fig. 1). The measured output signals of the system are obtained through the six piezo-sensors that are placed on the top surface of the funnel as shown in Fig. 2.

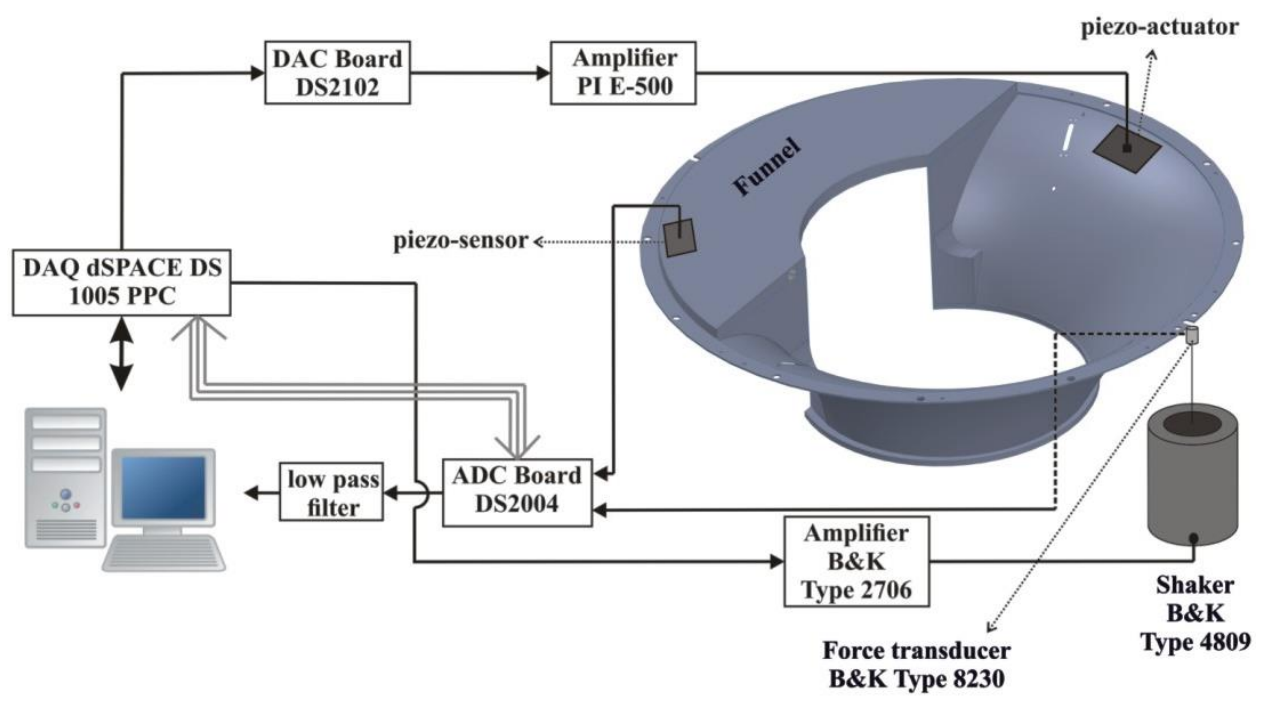

Fig. 2 Sketch of the experimental setup

dSPACE digital data acquisition (DAQ) with DS1005 PPC board is employed to compile the simulated disturbance and the designed control system in real-time analysis. The connection of the DAQ system with the actuators/sensors and the mainframe is provided by an analog to digital converter (ADC dSPACE DS2004) and a digital to analog converter (DAC dSPACE DS2102). Due to the different working voltage of dSPACE DAQ $( \pm 10 \mathrm{~V})$ compared to the transducers $([-60,200] \mathrm{V})$, an amplifier (PI E$500)$ is utilized to amplify the signal generated by DAQ with constant gain of 100 and similarly, the second amplifier with maximum input current up to $5 \mathrm{~A}$ (Brüel\&Kjær amplifier type 2706) is utilized to amplify the generated signal by DAQ system for the vibration exciter (Brüel\&Kjær shaker type 4809). The control law of the active vibration system is realized on the SIMULINK platform and then compiled regulator law with standard fixed step explicit ODE5 solver (Dormand-Prince method [31]) is downloaded to the dSPACE DAQ in real-time by means of experimental rig shown in Fig. 3. 




Fig. 3 Experimental rig of the closed-loop system

The simulated disturbance acts on the structure by the shaker which is connected to the funnel by a force transducer (Brüel\&Kjær DeltaTron Type 8230-001) with the sensitivity of $22 \mathrm{mV} / \mathrm{N}$. This transducer measures the mechanical force applied to the structure that is used as the definition of the uncertainty weighting function of input disturbance. Furthermore, it should be mentioned that in the rest of the paper, due to the computational limitations, only four sets of actuators and sensors $\left(L_{1}, L_{2}, R_{1}\right.$, and $\left.R_{2}\right)$ are activated for the modeling and control design purposes.

\section{SYSTEM IDENTIFICATION}

In this research, predictive error minimization (PEM) subspace identification algorithm [26] in the time domain for continuous LTI systems is utilized to estimate the system matrices from input-output data of the mechanical configuration shown in Fig. 2. The process begins with collecting data (voltage) from the input/output channels of the open-loop experimental setup. This includes the external input mechanical excitation channel that is realized by shaker $\left(B_{w}\right)$ and the input piezoelectrical excitation channel for applied control effort by means of functional modules $(B)$. Accordingly, the reduced order deterministic MIMO LTI system in the state-space framework which represents the dynamics of the funnel structure with distributed piezo-transducers in a constraint frequency range can be constructed through the trust-region-reflective Newton technique as $[27,32-34]$

$$
\begin{aligned}
& \dot{x}=A x+B u+B_{w} d, \\
& y=C x+D u+w_{n},
\end{aligned}
$$

where $x \in R^{n}, u \in R^{m}, d \in R^{p}$, and $y \in R^{q}$ are the state, control input, disturbance input, and output vectors, respectively. In addition, $A, B, B_{w}, C$, and $D$ correspondingly represent 
the matrices of states, control inputs, input disturbances, outputs, and feedforward. Furthermore, $w_{n}$ stands for the measurement noise which is considered to be an uncorrelated zero-mean Gaussian stochastic process with constant power spectral density $\left(W_{n}\right)$ so that $E\left\{w_{n} w_{n}{ }^{T}\right\}=W_{n} \delta(t-\tau)$, in which $E$ is the expectation operator [29].

\section{4. $\mu$-SYNTHESIS}

The coupled piezo-laminated funnel shape structure as shown in Fig. 4 has five inputs including one disturbance channel and four actuation signals. The measured output of the system consists of four channels associated with four piezo-sensors bounded on the structure. The modeling uncertainty for the input matrix and input disturbance matrix are assumed to be multiplicative unstructured uncertainty [35].



Fig. 4 Closed-loop system with multiplicative uncertainty

In Fig. $4 K(s)$ the controller is represented as designed on the basis of the output feedback $\mu$-synthesis. $n$ stands for the white Gaussian output measurement noise, respectively. In addition, $W_{\text {dist }}, W_{R d i s t}, W_{n}$, and $W_{R i}(i=1,2,3,4)$ are the frequency dependent norm-bounded weighting function for external input disturbance signal, the multiplicative uncertainty of disturbance, a high pass filter representing the activation frequency of the noise signal, and weighting function of the actuation uncertainty, respectively. Also, as a standard multiplicative representation, it is assumed that $\left\|\Delta_{\text {dist }}\right\|_{\infty} \leq 1$ and $\left\|\Delta_{\text {act } i}\right\|_{\infty} \leq 1, i=1, \ldots, 4$ [36]. For the sake of applying the structured singular value theory to the plant under study, the signal configuration in Fig. 4 should be recast into linear fractional transformation (LFT) [37] representation that is shown in the standard form in Fig. 5. 


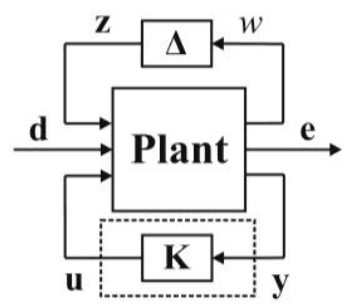

Fig. 5 LFT representation of the control system

In Fig. 5, the plant is an open-loop interconnection which encloses certain elements such as the nominal structure model and all the weighting functions. $\Delta$-block parameterizes all of the defined uncertain elements in the previous representation of Fig. 4. The inputs to the MIMO plant are allocated into three standard groups in which $w$ represents the perturbation and, similarly, the outputs are grouped into three signals with $e$ representing the error signal. The LFT representation covers the set of all the control systems $\mathrm{F}_{U}$ so that $\left\{\mathrm{F}_{U}(P(s), \Delta): \max _{\omega} \bar{\sigma}[\Delta(\mathrm{j} \omega) \leq 1]\right\}$ with $\omega$ being the frequency so that for perturbation $\Delta$, stabilizing controller $K(s)$ exists that satisfies the following equation:

$$
\left\|\mathrm{F}_{L}\left[\mathrm{~F}_{U}(P(s), \Delta), K(s)\right]\right\|_{\infty}=\left\|\mathrm{F}_{U}\left[\mathrm{~F}_{L}(P(s), K(s)), \Delta\right]\right\|_{\infty}<1 .
$$

For given controller $K(s)$, constrained $H_{\infty}$-index function of Eq. (2) with the predefined augmented uncertainty structure of LFT representation in Fig. 5 can be checked by examining the structured singular values of the closed-loop system if and only if $K(s)$ satisfies the following constraint over frequency $\omega$

$$
\max \mu_{\Delta}\left[\mathrm{F}_{L}\left[\mathrm{~F}_{U}(P(s), \Delta), K(s)\right]\right]<1 .
$$

The standard $\mu$-synthesis minimizes the maximum structured singular value of the close-loop system over frequency: $\min \left\{\max \left[\mu_{\Delta}\left[\mathrm{F}_{L}\left[\mathrm{~F}_{U}(P(s), \Delta), K(s)\right]\right](j \omega)\right]\right.$, stabilizing $\left.K(s)\right\}$. In order to solve the $\mu$-synthesis problem, $\mu_{\Delta}$ should be replaced with the upper bound.

Definition 1 [38] Considering $M$ to be a constant matrix and the uncertainty $\delta$, the upper bound of $\mu_{\delta}(M)$ is defined as inf $\bar{\sigma}\left(D M D^{-1}\right)$ for $D \in D_{\delta}$ with $D_{\delta}$ being the set of all matrices $D$ that satisfy $D \delta=\delta D$.

Using Definition 1 for the optimization function of the $\mu$-synthesis and $\Delta$, the optimization equation is reformatted as

$$
\min _{K(s)} \max _{\omega} \min _{D_{\omega} \in D_{\Delta}} \bar{\sigma}\left[D_{\omega} F_{L}(P(s), K(s))(j \omega) D_{\omega}^{-1}\right]
$$

by defining $D$ as a frequency dependent function that satisfies $D_{\omega} \in D_{\Delta}$ for an arbitrary frequency $\omega$, and replacing $\max _{\omega} \bar{\sigma}\left[\right.$.] with $\|.\|_{\infty}$, the optimization problem in Eq. (4) can be rewritten as

$$
\min _{K(s)} \min _{D, D_{\omega} \in D_{\Delta}}\left\|D F_{L}(\mathrm{P}(s), \mathrm{K}(s))(j \omega) D^{-1}\right\|_{\infty},
$$

then, assuming $U$ as a block orthogonal complex matrix for complex $M$ (Definition 1) to have the same structure as $D \in D_{\Delta}$ and satisfying $U^{*} U=U U^{*}=I$ with $I$ being the identity 
matrix and superscript * denoting the complex conjugate of the original matrix, it is easy to obtain $\bar{\sigma}\left[(U D) M(U D)^{-1}\right]=\bar{\sigma}\left[D M D^{-1}\right]$. This is due to the fact that the matrix multiplication with orthogonal matrix does not change $\bar{\sigma}$ and, as a result, one can transform $D$ into $U D$ without changing the maximum structured singular value of the system. By the introduction of an appropriate $U$, it is possible to restrict $D_{\omega}$ to be real-rational, stable, and minimum-phase transfer function symbolized as $\widehat{D}(s)$ [39]. The optimization index, Eq. (5), based on this transformation and due to the orthogonality of the introduced matrix is reformatted as

$$
\min _{K(s)} \min _{\widehat{D}(s) \in D_{\Delta}}\left\|\hat{D} F_{L}(P(s), K(s))(j \omega) \hat{D}^{-1}\right\|_{\infty},
$$

The transformed optimization problem of Eq. (6), as shown in Fig. 6, can be solved iteratively by means of $D-K$ iteration approach [40, 41].

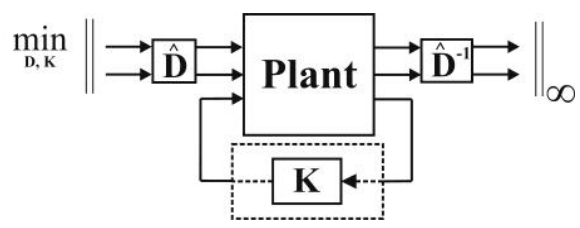

Fig. 6 Replacing $\mu_{\Delta}$ with the upper bound

\subsection{D-K iteration algorithm}

1. Solve optimization problem $\min _{K(s)}\left\|\hat{D} F_{L}(P(s), K(s))(j \omega) \widehat{D}^{-1}\right\|_{\infty}$, with an initial guess of stable, minimum-phase, and real rational $\bar{D}(s)$ and define $P_{D}(s)$ as shown in Fig. 7.

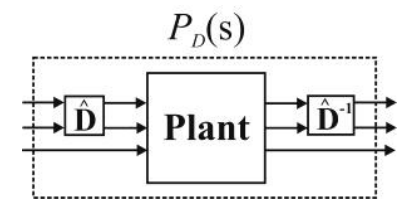

Fig. 7 Real-ration $D$ scaling in $D$ - $K$ iteration

2. With known $P_{D}(s)$ from the previous step, solve standard $H_{\infty}$ problem with the optimization function of $\min _{K(s)}\left\|\mathrm{F}_{L}(P(s), K(s))\right\|_{\infty}$.

3. With fixed controller $K(s)$ from the previous step, move the optimization over $D(s)$ and look for an appropriate frequency dependent function $D(s)$ in a certain range of frequency to satisfy the previous constraints on $\widehat{D}(s)$.

\section{EXPERIMENTAL AND NUMERICAL RESULTS}

In this section, the experimental investigation regarding the performance of the output feedback control system in suppressing the vibration of the MRI funnel structure is studied in detail. Considering the amount of variables that are involved in the modeling of the 
structure and the control system and keeping in view our limitations on computation power, we shall confine our attention to a systematic procedure in evaluating the closed-loop system. For this purpose, first a reduced order model of the real structure with distributed piezoelectric sensor/actuator groups is obtained by means of system identification. In particular, a MIMO LTI system in state-space representation of the structure including the dynamics of the sensors/actuators is estimated in the time domain by utilizing the MATLAB System Identification toolbox. The reduced order model is assumed to cover the dynamics of the system within the frequency range of $0-18 \mathrm{~Hz}$ which includes two natural frequencies of the structure $(9.72 \mathrm{~Hz}$ and $15.22 \mathrm{~Hz})$. In order to present the identification quality, the frequency response function (FRF) of the real structure is compared with bode diagram of the identified model in the nominal frequency range of Fig. 8(a, b, and c). It is worth mentioning that the reduced order model is a sixth order LTI object. The identified system is employed to design a robust controller on the feedback channel based on the output measurements that are collected by the four piezo-sensors $\left(L_{1}, L_{2}, R_{1}\right.$, and $\left.R_{2}\right)$ and dSPACE DS2004 based on the $D-K$ iteration approach that is explained in section 4 . Based on the FRF of the system, appropriate weighting functions are selected on the input channels that represent the frequency dependent uncertainty bounds and activation frequency of each channel.


Fig. 8a Comparison of FRF of the real structure with the identified model for disturbance channel 



Fig. 8b Comparison of FRF of the real structure with the identified model for actuator groups $L_{1}$ and $L_{2}$ 

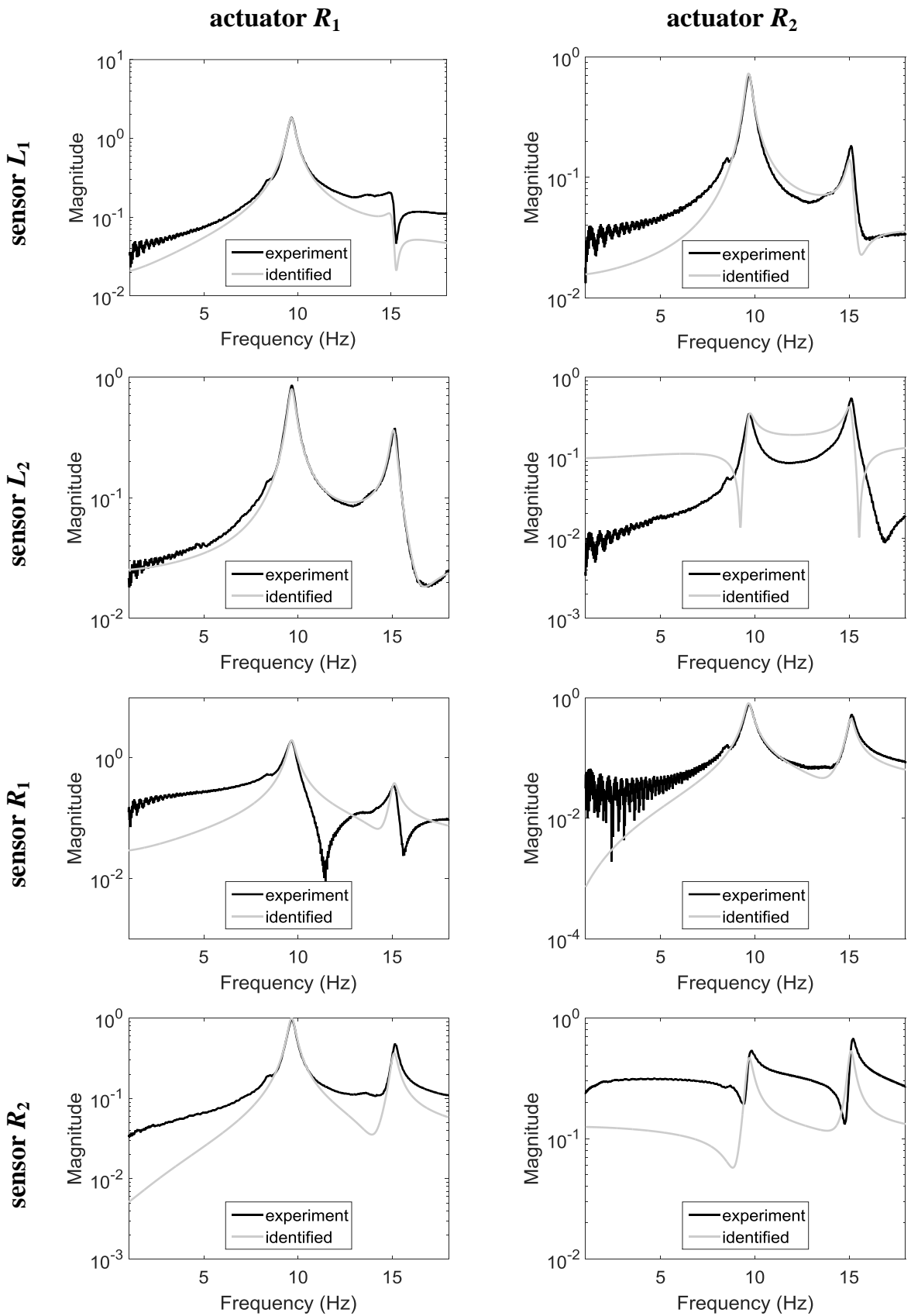

Fig. 8c Comparison of FRF of the real structure with the identified model for actuator groups $R_{l}$ and $R_{2}$ 
Due to the multiplicative nature of the augmented uncertainty, the weighting functions are selected as shown in Fig. 9 to cover the higher order mode-shapes of the structure which are neglected in the modeling procedure.

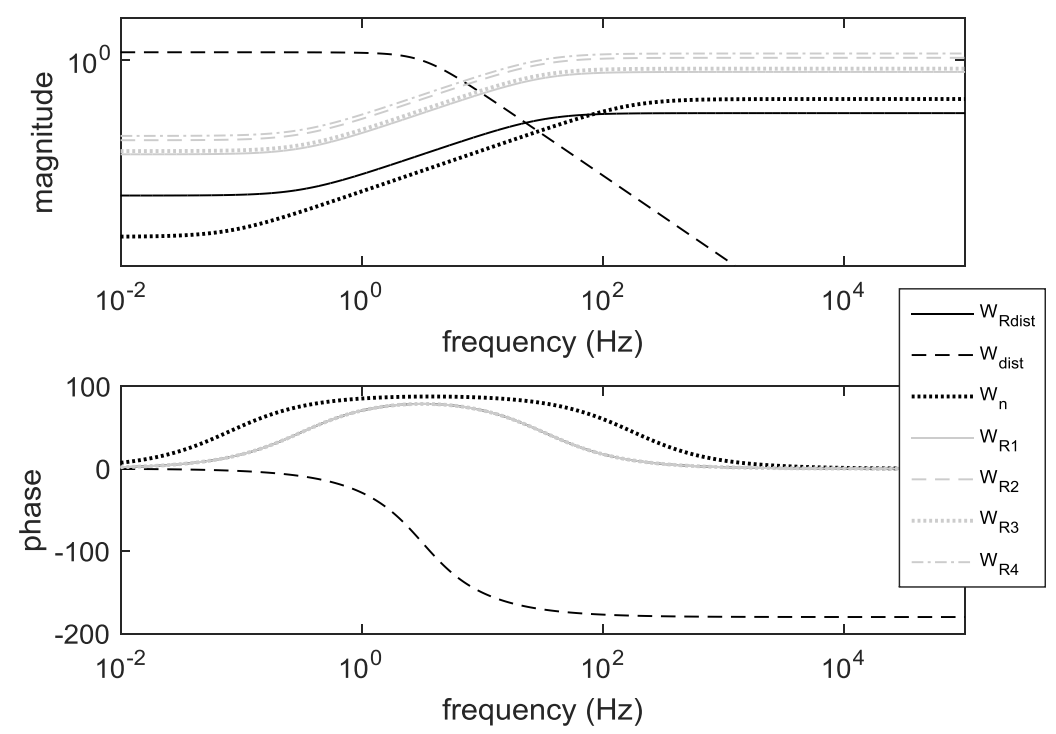

Fig. 9 Weighting functions associated with uncertainty presentation in control design

In order to investigate the performance of the output feedback control system, the structure is excited through the disturbance channel by a sweep sine signal with a frequency range of $0-18 \mathrm{~Hz}$ for both the open loop and closed-loop systems. The applied mechanical disturbance signal is measured by the force transducer (B\&K 8230) and represented in Fig. 10. Accordingly, the output measurements are obtained by the piezosensors and are depicted in Fig. 11. Fig. 11 compares the transient response of the open loop system and the closed-loop system based on the designed controller. It can be observed that the $\mu$-control system has successfully alleviated the vibration amplitude although the external disturbance continues to excite the structure.

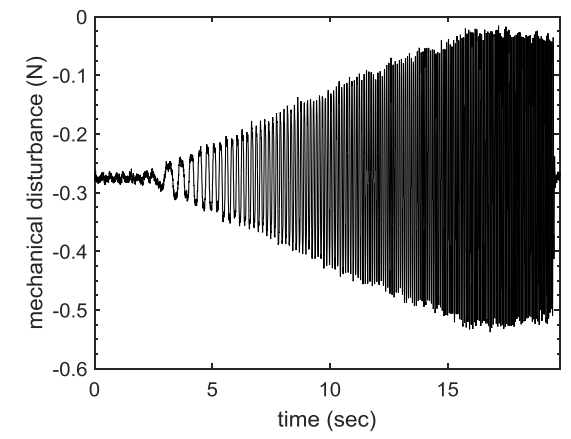

Fig. 10 The amplitude of applied mechanical disturbance through shaker 

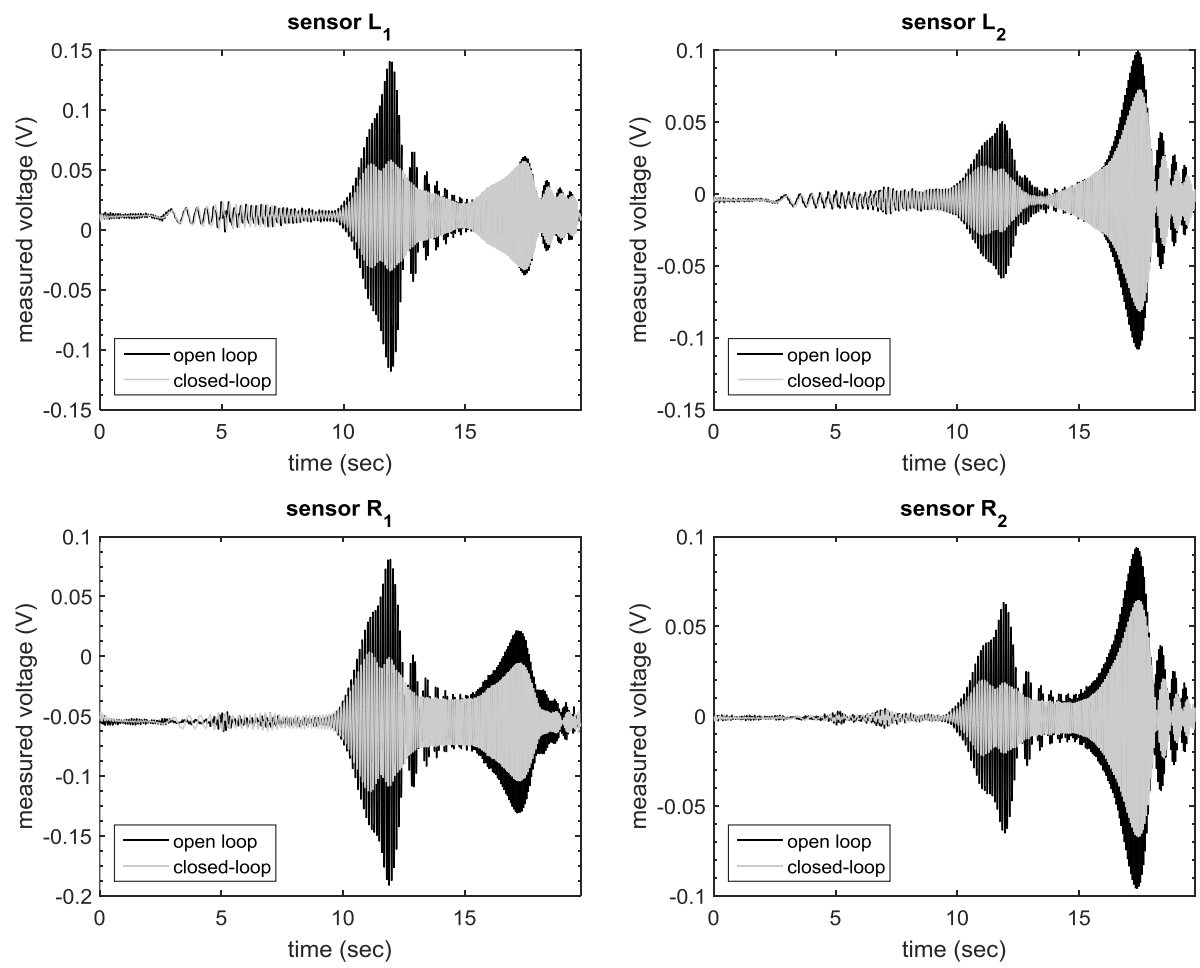

Fig. 11 The vibration suppression performance of the control system in the nominal frequency range

The applied control effort for the sweep sine signal is presented in Fig. 12. As expected, the control voltage for the $L_{2}$ and $R_{2}$ actuator groups are less than for the $L_{l}$ and $R_{l}$ groups which are due to the small level of strain energy that reaches to these actuators because of the distance between the disturbance source and the actuator elements (see Figs. 1 and 2).


Fig. 12 Applied control effort on the piezo-actuator groups 
In addition, by the introduction of the appropriate weighting functions (see Fig. 9), that contain the frequency dependent information of the actuator elements, the applied control efforts have a smooth behavior with the maximum amplitude of $55 \mathrm{~V}$.

In order to investigate the spillover effect of the controller, the Fast Fourier Transformation (FFT) analysis was performed on the control signal to clarify the frequency content of the applied voltage [42]. The result as depicted in Fig. 13 shows that although the disturbance is acting within the maximum frequency of $18 \mathrm{~Hz}$, the applied control effort of actuator $R_{l}$ leads to excitation of higher order mode-shape. However, significant reductions in the magnitude of the applied control effort can be observed for all of the actuation elements due to the introduction of the weighting functions. In addition, in the mechanical systems, the lower eigenfrequencies have a dominant impact, which is also confirmed here by the influence of the first, second, and third eigen-frequencies. Therefore, even if the high-frequency excitation would occur, it would not deteriorate the controller performance significantly due to the negligible influence of the higher modes with respect to the dominant mode-shapes.

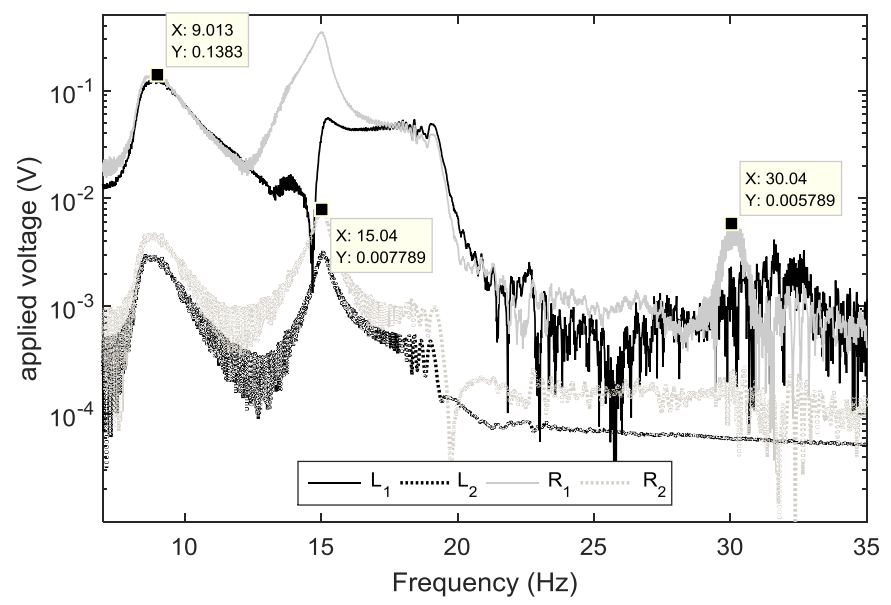

Fig. 13 FFT analysis on the control effort

Finally, by aiming at investigating the robust performance of the closed-loop system in stabilizing the unmodeled higher order dynamics of the MRI funnel structure, the coupled system is excited through the disturbance channel with a sweep sine signal that charges the higher order mode-shapes of the structure in the frequency range of 20-38 Hz. The applied mechanical force acts on the system within $30 \mathrm{sec}$ and the experimental results for the output measurements are presented in Fig. 14. In this figure, the controlled and the uncontrolled cases are brought together in four subplots to evaluate the robustness of the control system. It can be observed that the attenuation performance is reduced compared to the nominal frequency range but the control system mostly suppressed the vibration. Furthermore, as it can be seen in Fig. 15, the applied control effort on actuator $R_{l}$ saturates for a short period between 6.8 and $7.7 \mathrm{sec}$ which leads to performance loss that is mostly detected in sensor elements $L_{l}$ and $R_{l}$. This time period stands for the higher order unmodeled dynamics with the natural frequency around $27 \mathrm{~Hz}$. 



Fig. 14 Robust vibration attenuation performance for unmodeled dynamics

By comparing the control effort in the nominal frequency range (Fig. 12) and the highfrequency area (Fig. 15), it can be seen that the control system loses its optimality since by using more actuation energy less performance is achieved [42]. This emphasizes the importance of the nominal system order and the weighting functions in the control design procedure.
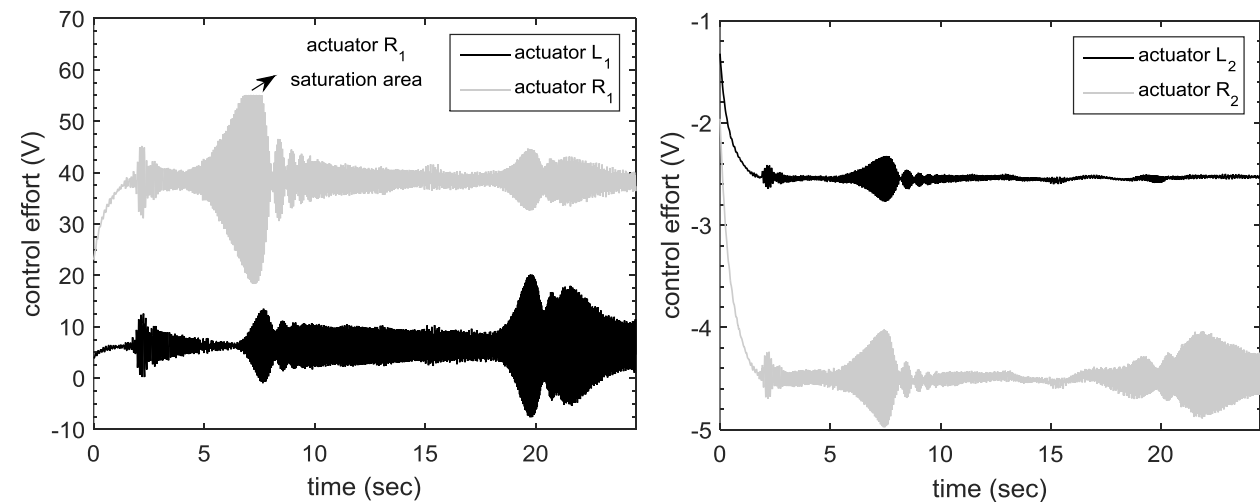

Fig. 15 Applied voltage on the piezo-actuators in high-frequency range 


\section{CONCLUSION}

In this paper, the robust vibration control performance of the funnel-shaped structure that is used in MRI devices is evaluated experimentally. The problem of structural vibration and acoustic noise in the MRI scanners is an important challenge in the development of the technology in these devices that can be addressed properly by active vibration control and active noise control. In order to relax the limitations on the exact modeling of the dynamics of the structure, it is crucial to design regulators that can handle the modeling uncertainty. It has been proven that with limited energy consumption, highquality vibration suppression can be achieved by a combination of appropriate uncertainty modeling and robust controller design.

\section{REFERENCES}

1. Yao, G.Z., Mechefske, C.K., Rutt, B.K., 2005, Vibration analysis and measurement of a gradient coil insert in a $4 T$ MRI, Journal of Sound and Vibration, 285, pp. 743-758.

2. Rutt, B.K., 2003, Safety issues in MRI, The 11th Scientific Meeting of the International Society for Magnetic Resonance in Medicine, Toronto, Canada.

3. Mechefske, C.K., Geris, R., Gati, J.S., Rutt, B.K., 2002, Acoustic noise reduction in a 4 T MRI scanner, Magnetic Resonance Materials in Physics, Biology and Medicine, 13, pp. 172-176.

4. Turner, R., 1993, Gradient coil design: a review of methods, Magnetic Resonance Imaging, 1, pp. 903-920.

5. Katsunuma, A., Takamori, H., Sakakura, Y., Hamamura, Y., Ogo, Y., Katayama, R., 2002, Quiet MRI with novel acoustic noise reduction, Magnetic Resonance Materials in Physics, Biology and Medicine, 13, pp. 139-144.

6. Edelstein, W.A., Mallozzi, R.P., Hedeen, R.A., El-Hamamsy, S.-A., Miller, M.L., Thompson, P.S., Ackermann, R.A., Amm, B.C., Fura, J.P., Radziun, M.J., Dean, D.E., Mansell, S.T., Purgill, D.A., Vavrek, R.M., 2002, Low noise MRI scanner, US 6437568 B1, Grant Application number US 09/676,945.

7. Wu, Y., Chronik, B.A., Bowen, C., Mechefske, C.K., Rutt, B.K., 2000, Gradient-induced acoustic and magnetic field fluctuation in a 4T whole-body MR imager, Magnetic Resonance in Medicine, 44, pp. 532-536.

8. McJury, M., Stewart, R.W., Crawford, D., Tomas, E., 1997, The use of active noise control (ANC) to reduce acoustic noise generated during MRI scanning: some initial results, Magnetic Resonance Imaging, 15(3), pp. 319-322.

9. Park, S.H., Kim, H., Chung, S.C., Chung, S.T., Chung, Y., Moon, C.W., Sin, C.H., Wong, E.K., Cho, Z.H., 1997, Analysis of acoustic noise in MRI, Magnetic Resonance Imaging, 15(7), pp. 815-822.

10. Price, D.L., De Wilde, J.P., Papadaki, A.M., Curran, J.S., Kitney, R.I., 2001, Investigation of Acoustic Noise on 15 MRI Scanners from 0.2 T to 3 T, Journal Of Magnetic Resonance Imaging, 13, pp. 288-293.

11. Edelstein, W.A., Hedeen R.A., Mallozzi, R.P., El-Hamamsya, S.-A., Ackermann, R.A., Havens, T.J., 2002, Making MRI Quieter, Magnetic Resonance Imaging, 20, pp. 155-163.

12. Mechefske, C.K., Geris, R., Gati, J.S., Rutt, B.K., 2002, Acoustic noise reduction in a 4 T MRI scanner, Magnetic Resonance Materials in Physics, Biology and Medicine, 13, pp. 172-176.

13. Oveisi, A., Gudarzi, M., Mohammadi, M.M., Doosthoseini, A., 2013, Modeling, identification and active vibration control of a funnel-shaped structure used in MRI throat, Journal of Vibroengineering, 15(1), pp. 1392-8716.

14. Mechefske, C.K., Shao, W., 2007, Analytical modeling of noise in MRI scanners, Proceedings of the ASME International Design Engineering Technical Conferences and Computers and Information in Engineering Conference, pp. 307-311.

15. Pribil, J., Pribilova, A., Frollo, I., 2012, Analysis of spectral properties of acoustic noise produced during magnetic resonance imaging, Applied Acoustics, 73, pp. 687-697.

16. Hurwitz, R., Lane, S.R., Bell, R.A., Brant-Zawadzki, M.N., 1989, Acoustic analysis of gradient-coil noise in MR imaging, Radiology, 173, pp. 545-548.

17. Qiu, J., Tani, J., 1995, Vibration control of a cylindrical shell used in MRI equipment, Smart Materials and Structures, 4, pp. 75-81. 
18. Nestorović, T., Koppe, T.H., Gabbert, U., 2005, Active vibration control using optimal LQ tracking system with additional dynamics, International Journal of Control, 78(15), pp. 1182 - 1197.

19. Nestorović, T., Koppe, T.H., Gabbert, U., 2006, Vibration control of a funnel-shaped shell structure with distributed piezoelectric actuators and sensors, Smart Materials and Structures, 15(4), pp. 1119-1132.

20. Jaensch, M., Lamperth, M.U., 2007, Development of a multi-degree-of-freedom micro positioning, vibration isolation and vibration suppression system, Smart Materials and Structures, 16, pp. 409-417.

21. Roozen, N.B., Koevoets, A.H., den Hamer, A.J., 2008, Active vibration control of gradient coils to reduce acoustic noise of MRI systems, IEEE/ASME Transactions on Mechatronics, 13(3).

22. Li, M., Lim, T.C., Lee J.H., 2008, Simulation study on active noise control for a 4-T MRI scanner, Magnetic Resonance Imaging, 26, pp. 393-400.

23. Oveisi, A., Gudarzi, M., 2015, Application of auto-tuning Ziegler-Nichols PID control in chaotic vibration suppression of a beam with nonlinear boundary conditions, Latin American Applied Research, 45, pp. 179-184.

24. Oveisi, A., Gudarzi, M., 2013, Adaptive sliding mode vibration control of a nonlinear smart beam: a comparison with self-tuning Ziegler-Nichols PID controller, Journal of Low Frequency Noise, Vibration and Active Control, 32(1+2), pp. 41-62.

25. Kerschena, G., 2006, Past, present and future of nonlinear system identification in structural dynamics, Mechanical Systems and Signal Processing, 20(3), pp. 505-592.

26. Oveisi, A., Nestorović, T., 2016, Robust observer-based adaptive fuzzy sliding mode controller, Mechanical Systems and Signal Processing, DOI: 10.1016/j.ymssp.2016.01.015.

27. Ljung, L., 1999, System Identification: Theory for the User, Prentice Hall, New Jersey, USA.

28. Hasheminejad, S.M., Oveisi, A., 2015, Active vibration control of an arbitrary thick smart cylindrical panel with optimally placed piezoelectric sensor/actuator pairs, International Journal of Mechanics and Materials in Design, 12(1), pp. 1-16.

29. van Overschee, P., de Moor, B.L., 2012, Subspace identification for linear systems: theoryImplementation-Applications, Kluwer Academic Publishers Group, Dordrecht, The Netherlands.

30. Gabbert, U., Köppe, H., Nestorović, T., Seeger, F., 2003, Modelling, simulation and optimal design of lightweight structures, Proceedings of the Adaptronic Congress, Wolfsburg, Germany.

31. Shampine, L.F., 1986, Some Practical Runge-Kutta Formulas, Mathematics of Computation, 46(173), pp. $135-150$

32. Oveisi, A., Nestorović, T., 2014, Robust mixed $H_{2} / H_{\infty}$ active vibration controller in attenuation of smart beam, Facta Universitatis Series: Mechanical Engineering, 12(3), pp. 235-249.

33. Byrd, R.H., Schnabel, R.B., 1988, Approximate solution of the trust region problem by minimization over two-dimensional subspaces, Mathematical Programming, 40, pp. 247-263 .

34. Sorensen, D.C., 1997, Minimization of a large-scale quadratic function subject to a spherical constraint, SIAM Journal on Optimization, 7(1), pp. 141-161.

35. Gudarzi, M., Oveisi, A., Mohammadi, M.M., 2012, Robust active vibration control of a rectangular piezoelectric laminate flexible thin plate: An LMI-based approach, International Review of Mechanical Engineering, 6(6), pp. 1217-1227.

36. Gudarzi, M., Oveisi, A., 2014, Robust control for ride comfort improvement of an active suspension system considering uncertain driver's biodynamics, Journal of Low Frequency Noise Vibration and Active Control, 33(3), pp. 317-340.

37. Limebeer, D.J.N., Green, M., 2010, Linear robust control, Dover Books on Electrical Engineering, Dover Publications.

38. Gu, D.-W., Petkov, P., Konstantinov, M.M, 2013, Robust control design with MATLAB, Advanced Textbooks in Control and Signal Processing, Springer-Verlag, London, UK.

39. Packard, A., Doyle, J.C, Balas, G., 1993, Linear multivariable robust control with a $\mu$ perspective, ASME Journal of Dynamic Systems, Measurement and Control, 115(2), pp. 310-319.

40. Lin, F., 2007, Robust control design: an optimal control approach, 1st edition, Wiley-Interscience.

41. Gudarzi, M., 2015, $\mu$-Synthesis Controller Design for Seismic Alleviation of Structures with Parametric Uncertainties, Journal of Low Frequency Noise, Vibration and Active Control, 34(4), pp. 491-512.

42. Oveisi, A., Shakeri, R., 2016, Robust reliable control in vibration suppression of sandwich circular plates, Engineering Structures, 116, pp. 1-11. 